\title{
Perimeter-Control Architecture for Optical Phased Arrays and Metasurfaces
}

\author{
Artur Davoyan ${ }^{1,2, *}$ and Harry Atwater ${ }^{1, \dagger}$ \\ ${ }^{1}$ Thomas J. Watson Laboratories of Applied Physics, California Institute of Technology, Pasadena, \\ California 91125, USA \\ ${ }^{2}$ Department of Mechanical and Aerospace Engineering, University of California, Los Angeles, 420 Westwood \\ Plaza, Los Angeles, California 90095, USA
}

(Received 3 May 2020; revised 10 July 2020; accepted 13 July 2020; published 14 August 2020)

\begin{abstract}
Tunable optical phased arrays and metasurfaces play an important role in a diverse range of applications from imaging and remote sensing to communications and displays. However, as the number of tunable elements grows, the required control architecture becomes insurmountably complex. Here, we discuss the concept of perimeter-controlled tuning to shape far-zone radiation. We discuss applications of our approach to beam forming, holography, and image projection. We show that, with a proper design, the complexity of a control architecture may be dramatically simplified. We further discuss the use of our method to time-sharing image projection and holography. Our concept is applicable to a variety of systems, including phased array optical antennas and metasurfaces.
\end{abstract}

DOI: 10.1103/PhysRevApplied.14.024038

\section{INTRODUCTION}

Arrays of coherent emitters, such as phased array antennas [1-8] and, more recently, metasurfaces [9-20], play an important role in a broad range of applications from ranging [4] and remote sensing [21] to free-space communications [22,23] and holography [12,24,25]. In particular, optical phased arrays, which are counterparts of rf antenna arrays [1,26-29], offer a compact and efficient solution for LIDAR [4-6] that is critical for autonomous navigation. Furthermore, such systems are of great promise for high-bandwidth in-space communication between satellites and deep space probes [22,23]. Recent progress with metasurfaces [9], which are arrays of synchronized subwavelength scatterers, offers opportunities for holography [25] and flat optics [9]. Finally, we note developments in phased array microwave and ultrasound systems for wireless power transfer $[30,31]$ and for $5 \mathrm{G}$ communications [26-29].

Among many challenges that such systems face, emergent phenomena associated with size scaling become increasingly important. Indeed, a simple estimate shows that a near-infrared $(\lambda \simeq 1 \mu \mathrm{m})$ array with a millimetersize aperture would consist of millions of emitters (see Appendix B for more details), all of which would have to interfere coherently to produce a desired far-field radiation pattern. Although the design and fabrication of very large passive arrays have been successfully demonstrated

\footnotetext{
*davoyan@seas.ucla.edu

†haa@caltech.edu
}

in recent years [32], tunable control of each element of the array presents a significant engineering challenge $[4,5,16,17]$. Indeed, with millions of elements in the array, simultaneous and independent addressing of each emitter would require complex control architectures beyond the reach of current technology. Further progress requires efficient control solutions to tune the phase or amplitude response of individual elements across the entire array.

Previously, several studies have considered row-column addressing to reduce the number of control elements for beam formation with planar rectangular phased arrays [33-36]. Specifically, nonlinear signal mixing [34,35] and interemitter coupling [35] schemes were considered. These methods, however, are not directly applicable to optical frequencies, where nonlinear responses are typically much weaker. Recently, in Ref. [37], an interesting row-column addressing scheme was proposed to steer an optical phased array. In particular, the radiation of each of the array elements was formed by an interference between respective column and row optical waveguide feeds. However, based on optical interference, this method is inherently limited, since the emitter amplitude depends on the feed phase (hence, there is the need for nonlinearity [33-36]), which significantly limits the functionality and class of operations that can be performed [1]. Notably, all previous works [33-37] consider column-row phase tuning of input feed waves. That is, control is achieved by engineering the carrier input. At the same time, recent advances with metasurfaces and phased arrays [10-17] suggest that phase tuning can also be attained at the individual element level (e.g., by an applied voltage). In this case, one may 
consider a column-row addressing of control signals, rather than column-row tuning of input carrier feed implemented earlier [33-37]. Such a control architecture has not been discussed previously.

Here, we introduce and discuss theoretically a perimetercontrol architecture for large area tunable phased array systems and phase gradient metasurfaces. In contrast to previous works, we consider column-row addressing of control signals with phase tuning at the individual element level. This allows us to attain a linear and phase-only tuning across the entire system. We discuss physical and mathematical implications of the proposed control scheme. We show that such a control scheme may offer efficient ways for beam steering and holography. We further elucidate fundamental relations between phased array optics, metasurfaces, and holography.

We begin our analysis with a general scenario of radiation formed by an array of electrically small emitters, i.e., radiation sources, which can be individual antennas in the case of a phased array antenna [1] or individual scatterers in the case of a metasurface [9]. We assume that each elementary source located at points $\mathbf{r}_{0 v}$ is excited with an amplitude $\mathbf{a}_{v}$ and a relative phase $\psi_{\nu}$ [see Fig. 1(a) and Appendix $\mathrm{C}] ; v$ indexes array elements. In this case, the radiated electric field in the far radiation zone of an array is given by a superposition of fields radiated by each source [1]:

$$
\mathbf{E}=\sum_{\nu} e^{-i \mathbf{k r}_{0 v}} \mathbf{a}_{v} e^{i \psi_{\nu}}
$$

Here, we employ a far-zone approximation assuming that $\mathbf{k r} \rightarrow \infty$ and $\mathbf{r} \gg D$, i.e., the distance to the observation point at $\mathbf{r}$ is much larger than the physical dimension, $D$, of the array; $\mathbf{k}$ is a free-space wavevector $|\mathbf{k}|=2 \pi / \lambda$. According to the expression above the far-zone radiation pattern is formed due to an interplay of two factors: array geometry, described by the factor $e^{-i \mathbf{k r}_{0 v}}$, and intrinsic property of each emitter defined by its complex amplitude and phase $\mathbf{a}_{v} e^{i \psi_{\nu}}$. Without loss of generality, we assume here a square array with $N \times N$ uniformly spaced elements, see Fig. 1(a). We note that the concept and principles discussed here may be extended to other array topologies, including circular and hexagonal element arrangements.

For a uniform spacing of array elements [Fig. 1(a)], the geometric phase $\mathbf{k r}_{0 v}$ factorizes into $\mathbf{k r}_{0 v}=k\left[l \Delta r_{x} \sin (\theta)\right.$ $\left.\cos (\phi)+n \Delta r_{y} \sin (\theta) \sin (\phi)\right]$, where $\Delta r_{x}$ and $\Delta r_{y}$ denote distances between array elements along $x$ and $y$ directions, and $l$ and $n$ enumerate array columns (i.e., discrete $x$ coordinate) and rows (i.e., discrete $y$ coordinate), respectively; $\theta$ and $\phi$ are azimuth and polar angles in the direction of observation [see Fig. 1(a)]. The radiated field in the far-zone is then expressed as

$$
\begin{aligned}
\mathbf{E}=\sum_{l} \sum_{n} \mathbf{a}_{l n} e^{i \psi_{l n}} \exp \left[-i k l \Delta r_{x} \sin (\theta) \cos (\phi)\right. \\
\left.-i k n \Delta r_{y} \sin (\theta) \sin (\phi)\right] .
\end{aligned}
$$

In a most generic scenario of a tunable system, each emitter is controlled independently by an applied control signal $V_{\nu}$. These control signals may correspond to an applied (a)

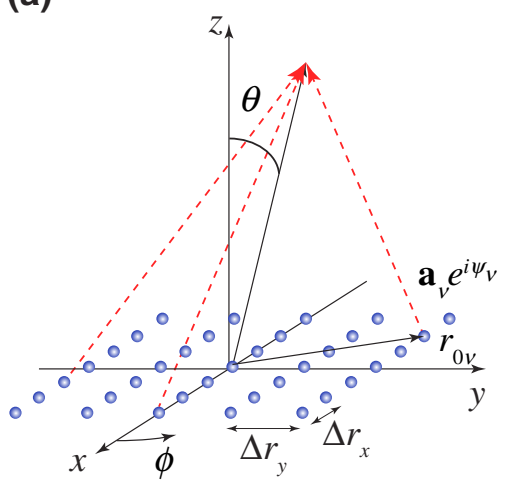

(b)

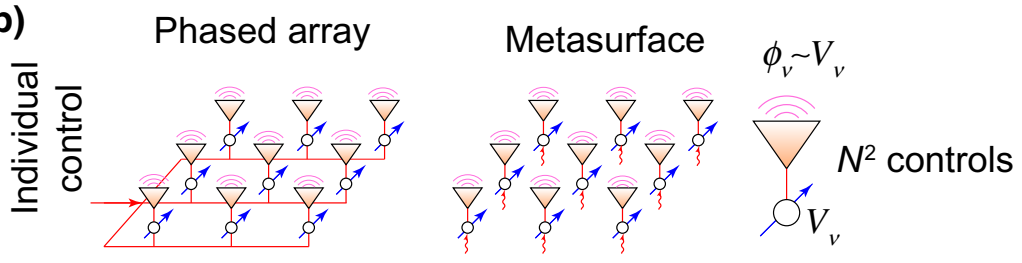

(c)

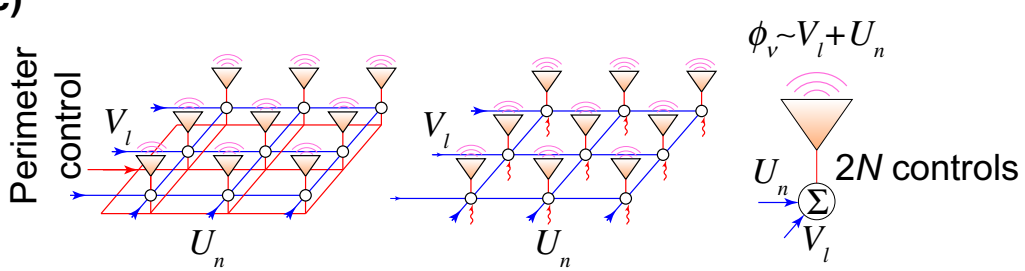

FIG. 1. (a) Schematic illustration of a planar array of emitters. Emitters can be either individual antennas of a phased array antenna or scatterers in the case of a metasurface. Emitters interact coherently to form a pattern in the far radiation zone. (b) Types of coherent arrays and associated control architectures. In phased array antennas, elements are fed with a common source that is then distributed to individual antennas via a network of phase shifters [2-6]. Metasurface elements, in contrast, are excited in parallel with an incident wave [9-17]. In both of these cases, conventional control architecture assumes that each element is tuned independently of its neighbors [2-6,16,17], thus, necessitating $N^{2}$ control signals (i.e., a control signal per array element). (c) Schematic illustration of a perimetercontrol architecture concept. Phase of each element of the array is defined by the superposition of control signals applied to respective columns and rows of the array. Total number of controls is $2 N$. 
voltage, temperature, or magnetic field. A schematic illustration of conventional control architectures is shown in Fig. 1(b). A control signal $V_{v}$ applied to the $v$ th emitter (i.e., $v$ th element of the array located in column $l$ and row $n$ ) changes its radiation properties, which, in the case of an electrically small emitter, corresponds to a change in amplitude and phase [16,17], i.e., $\mathbf{a}_{v}=\mathbf{a}_{l n}\left(V_{l n}\right)$ and $\psi_{v}=\psi_{l n}\left(V_{l n}\right)$. Clearly, in a generic case of simultaneous and independent addressing of the array elements, $N \times N$ independent control signals would be required. Evidently, as the number of array elements grows (see Appendix B), the complexity of the control architecture grows dramatically as well. To reduce control complexity and decrease the number of signals needed, we consider the perimetercontrol architecture depicted schematically in Fig. 1(c). Specifically, here control signals are applied not to individual elements of the array, but to the rows and columns of the array [Fig. 1(b)]. In this case, the control signal at the $v$ th element of the array is a function of control signals applied to respective column $l$ and row $n, V_{v}=$ $f\left(V_{l}+U_{n}\right) ; V_{l}$ and $U_{n}$ denote control signals along $x$ and $y$ directions of the array, respectively. We stress here a distinct difference with Refs. [33-37], where the phase of the feed electromagnetic wave is tuned at input columns and rows. In contrast, we tune the control signal (i.e., voltage), which then controls the phase at a given element [16]. One of the possible implementations of this scheme is discussed in Appendix D. For the sake of simplicity and to elucidate key manifestations of the proposed control architecture, we assume that array elements are identical and consider that emitter amplitude is independent of the applied control signal, i.e., $\mathbf{a}_{v}=\mathbf{a} \equiv$ const. (Notably, in principle, in many existing implementations [16,17], the amplitude varies with an applied voltage and such a dependence is not a fundamental constraint, i.e., one may potentially devise a phase-only tunable element [15].) At the same time, the emitter phase, $\psi_{\nu}$, is free to vary with an applied signal and may be expressed as $\psi_{v}\left(V_{v}\right) \equiv F\left(V_{v}\right)=F\left[f\left(V_{l}+U_{n}\right)\right]$. For a monotonic dependence of phase with an applied control signal (which is typically obtained in experiments), we may require that $F\left[f\left(V_{l}+U_{n}\right)\right]=V_{l}+U_{n}$, i.e., $f(\cdot)=$ $F^{-1}(\cdot)$. In this case, the array radiation in the far-zone is given simply as $\mathbf{E}(\theta, \phi)=\mathbf{a} Z_{\text {perim }}(\theta, \phi)$, where

$$
\begin{aligned}
Z_{\text {perim }}(\theta, \phi)= & \sum_{l} \sum_{n} \exp \left(i V_{l}+i U_{n}\right) \\
& \times \exp \left[-i k l \Delta r_{x} \sin (\theta) \cos (\phi)\right. \\
& \left.-i k n \Delta r_{y} \sin (\theta) \sin (\phi)\right],
\end{aligned}
$$

is the array factor of our perimeter-controlled system, which is a phased array antenna or metasurface.

We may further write this expression in a more compact matrix form:

$$
Z_{\text {perim }}(\theta, \phi)=\langle e|\mathbf{v} \otimes \mathbf{u}| e\rangle,
$$

where $\otimes$ denotes an outer product between two linearly independent vectors $\mathbf{v}_{l}=\exp \left[i V_{l}-i k l \Delta r_{x} \sin (\theta) \cos (\phi)\right]$ and $\mathbf{u}_{n}=\exp \left[i U_{n}-i k n \Delta r_{y} \sin (\theta) \sin (\phi)\right]$ associated with the phase gradient along columns and rows of the array, and $|e\rangle \mid=(1, \ldots, 1, \ldots, 1)$ is a unitary vector. Therefore, the far field of such a perimeter-controlled array is given as a convolution of two linearly independent responses associated with the orthogonal directions of the array. Below, we discuss applications and limitations of such perimetercontrol architecture in the context of beam steering and holography.

\section{TUNABLE BEAM STEERING}

Beam forming and steering is one of the major areas of interest, where phased array optics, both antenna arrays and metasurfaces, are of a great promise $[2-6,16,17,19]$. In this case, phases of the individual radiation sources are superimposed coherently to form a narrow "pencil" beam [1]. The direction of emission may be controlled by tailoring the phase gradient distribution across the array [1]. For a uniform planar array, with an equidistant spacing of emitters, and for a linear gradient of phase between the elements, the array factor is found to be [1]

$$
\begin{aligned}
Z_{\text {pencil }}(\theta, \phi)= & \sum_{l} \exp i l\left[\Delta \psi_{x}-\frac{2 \pi}{\lambda} \Delta r_{x} \sin (\theta) \cos (\phi)\right] \\
& \times \sum_{n} \exp i n\left[\Delta \psi_{y}-\frac{2 \pi}{\lambda} \Delta r_{y} \sin (\theta) \sin (\phi)\right],
\end{aligned}
$$

where $\Delta \psi_{x}$ and $\Delta \psi_{y}$ are phase increments along the $x$ and $y$ directions of the array, respectively.

By comparing Eq. (5) with an expression for an array factor of the perimeter-control architecture, Eq. (3), it is trivial to show that $Z_{\text {perim }}(\theta, \phi) \equiv Z_{\text {pencil }}(\theta, \phi)$ when $\psi_{v}\left(V_{v}\right) \equiv V_{l}+U_{n}=l \Delta \psi_{x}+n \Delta \psi_{y}$. Obviously, this relation holds for $V_{l}=l \Delta \psi_{x}$ and $U_{n}=n \Delta \psi_{y}$. Therefore, by appropriately choosing gradients of control signals along columns and rows of the array, we reproduce the full beam-steering functionality that is attained in arrays with individually controlled elements. For a linear gradient of phase (or control signal in our case), the array factor, $Z$, convolves into the following compact expression [cf. Eq. (4)] [1]:

$$
Z(\theta, \phi)=\frac{\sin \left(\frac{N \delta_{x}}{2}\right)}{\sin \left(\frac{\delta_{x}}{2}\right)} \frac{\sin \left(\frac{N \delta_{y}}{2}\right)}{\sin \left(\frac{\delta_{y}}{2}\right)},
$$

where $\delta_{x}=\Delta \psi_{x}-(2 \pi / \lambda) \Delta r_{x} \sin (\theta) \cos (\phi)$ and $\delta_{y}=$ $\Delta \psi_{y}-(2 \pi / \lambda) \Delta r_{y} \sin (\theta) \sin (\phi)$ are array phase factors along $x$ and $y$ directions, respectively. The direction of the beam is found from the condition $\delta_{x}=\delta_{y}=0$ 
and yields $\Delta \psi_{x}=(2 \pi / \lambda) \Delta r_{x} \sin \left(\theta_{0}\right) \cos \left(\phi_{0}\right)$ and $\Delta \psi_{y}=$ $(2 \pi / \lambda) \Delta r_{y} \sin \left(\theta_{0}\right) \sin \left(\phi_{0}\right)$, where $\theta_{0}$ and $\phi_{0}$ are angular coordinates of the pencil beam (i.e., the direction of the main radiation lobe). Hence, by choosing to control the signal gradient along the perimeter, the desired phase gradient and associated beam steering may be achieved. Figure 2 shows several examples of perimeter-controlled beam steering. Here, we assume, as an example, a $10 \times 10$ array; however, our concept is applicable to arrays with millions of elements. To avoid grating lobes, the spacing between the array elements has to be less than the wavelength $[16,17]$. In our calculations, we consider $\Delta r_{x}=\Delta r_{y}=$ $\lambda / 4$. The beam width between two adjacent nulls along the $x$ and $y$ axes are found to be $\left(2 \lambda / \Delta r_{x} N\right)$ and $\left(2 \lambda / \Delta r_{y} N\right)$, respectively.

\section{PHASE GRADIENT FLAT OPTICS}

It is instructive to consider the case of a metasurface excited with an obliquely incident plane wave [9]. Consider, without loss of generality, a plane wave incident at some angle $\alpha$ on an infinitesimally thin metasurface located at the interface of two media with permittivities $\varepsilon_{1}$ and $\varepsilon_{2}$ (see Appendix A). Notably, here we assume an array with a finite aperture $D_{x} \times D_{y}=N \Delta r_{x} \times N \Delta r_{y}$, where $D_{x}$ and $D_{y}$ are physical sizes of the array in the $x$ and $y$ directions, respectively. The oblique incidence will induce an additional phase delay between excited metasurface elements $\Delta \psi_{x}^{\text {inc }}=k \sqrt{\varepsilon_{1}} \sin (\alpha) \Delta r_{x}$ (we assume that the wave is incident from a medium with permittivity $\varepsilon_{1}$ ). The radiation pattern of the array in either medium is defined by the array factor, Eq. (6), with modified array phase factors, $\delta_{x}=\Delta \psi_{x}+\Delta \psi_{x}^{\text {inc }}-k \sqrt{\varepsilon_{i}} \Delta r_{x} \sin (\theta) \cos (\phi)$ and $\delta_{y}=\Delta \psi_{y}-k \sqrt{\varepsilon_{i}} \Delta r_{x} \sin (\theta) \sin (\phi)$, where $\varepsilon_{i}=\varepsilon_{1}$ for the radiation pattern above the metasurface, i.e., "reflected" beam, and $\varepsilon_{i}=\varepsilon_{1}$ for radiation below the metasurface, i.e., "refracted" beam. Clearly, the array phase factors are determined by three contributions, namely, the oblique incidence, $k \sqrt{\varepsilon_{1}} \sin (\alpha) \Delta r_{x}$; the intrinsic phase gradient of the metasurface, $\Delta \psi_{x}$; and the array geometry, $-k \sqrt{\varepsilon_{i}} \Delta r_{x} \sin (\theta) \cos (\phi)$. The direction of emission is again found from the condition $\delta_{x}=\delta_{y}=0$. For given metasurface phase gradients $\Delta \psi_{x}$ and $\Delta \psi_{y}$ and incidence angle $\alpha$, we arrive at two coupled equations that determine the direction of emission:

$$
\begin{aligned}
& \sqrt{\varepsilon_{i}} \sin (\theta) \cos (\phi)=\sqrt{\varepsilon_{1}} \sin (\alpha)+\frac{\Delta \psi_{x}}{k \Delta r_{x}}, \\
& \sqrt{\varepsilon_{i}} \sin (\theta) \sin (\phi)=\frac{\Delta \psi_{y}}{k \Delta r_{y}},
\end{aligned}
$$

At the limit of $\Delta r_{x} \rightarrow 0$ and $\Delta r_{y} \rightarrow 0$, we arrive at expressions similar to those of generalized Snell's laws of reflection and refraction defined by phase gradients in $x$ and $y$ direction, $\partial \psi_{x} / \partial x$ and $\partial \psi_{y} / \partial y$, respectively [7]. Here, in our perimeter-control architecture, the phase increments $\Delta \psi_{x}$ and $\Delta \psi_{y}$ are linearly related to signals $V_{l}$ and $U_{n}$ applied on the perimeter of the array. Hence, our approach allows to fully control beam reflection and refraction. While we base our discussion on array theory [1], at the limit of $\Delta r_{x} \rightarrow 0$ and $\Delta r_{y} \rightarrow 0$ (i.e., as $N \rightarrow \infty$ for a fixed aperture size), we arrive at the familiar plane wave diffraction theory [37]. In this case, it is possible to show that these radiated fields are familiar reflected and refracted plane waves, i.e., $E \sim$ $\exp \left\{\mp i k \sqrt{\varepsilon_{i}}[\sin (\theta) \cos (\phi) x+\sin (\theta) \sin (\phi) y+\cos (\theta) z]\right\}$, where angles $\theta$ and $\phi$ are selected, according to Eq. (7). Therefore, actual generalized Snell's laws are recovered at this limit.

\section{PERIMETER-CONTROL HOLOGRAPHY}

Holography is another important application where metasurfaces and phased arrays play an important role [24]. Of particular interest are phase-only holograms [38] (also known as kinoforms), in which a desired image is reconstructed as a Fourier transform of a holographic phase-only mask, $H_{\text {phase }}\left(x^{\prime}, y^{\prime}\right) \equiv e^{i \psi\left(x^{\prime}, y^{\prime}\right)}$, i.e., $I(x, y)=$ $\mathcal{F}_{2 D}\left[H_{\text {phase }}\left(x^{\prime}, y^{\prime}\right)\right]$, where $I(x, y)$ denotes a complex amplitude of a scalar field in the image plane $(x, y)$ (not to be confused with field intensity) and $\mathcal{F}_{2 \mathrm{D}}(\cdot)$ is a twodimensional (2D) Fourier transform acting in the $\left(x^{\prime}, y^{\prime}\right)$ plane of the hologram [Fig. 3(a)]. A computer-generated hologram (i.e., a discrete phase mask) is then produced by discretization of the desired phase profile [38]: $e^{i \psi\left(x^{\prime}, y^{\prime}\right)} \simeq$ $\sum_{l, n} e^{i \psi\left(x^{\prime} l, y^{\prime}{ }_{n}\right)} \delta\left(x^{\prime}-x^{\prime}{ }_{l}, y^{\prime}-y_{n}^{\prime}\right)$, where $\delta(\cdot)$ denotes the Dirac delta function. In this case, the reconstructed image is well approximated by a discrete 2D Fourier transform of the discretized phase mask:

$$
\begin{aligned}
I(x, y) & =\mathcal{F}_{2 \mathrm{D}}\left[e^{i \psi\left(x^{\prime}, y^{\prime}\right)}\right] \simeq \mathcal{F}_{2 \mathrm{D}}\left[e^{i \psi\left(x^{\prime} l, y^{\prime}{ }_{n}\right)}\right] \\
& =\sum_{l, n} e^{i \psi\left(x^{\prime}{ }_{l}, y^{\prime}{ }_{n}\right)} e^{-i k\left(\frac{x}{z} x^{\prime}{ }_{l}+\frac{y}{z} y_{n}^{\prime}\right)}=I\left(x_{p}, y_{q}\right),
\end{aligned}
$$

here $l, n$ and $p, q$ enumerate corresponding columns and rows in the holographic mask and reconstructed image planes, respectively.

Recognizing that $k \sin (\theta) \cos (\phi)=k_{x}=k(x / z)$ and $k \sin (\theta) \sin (\phi)=k_{y}=k(y / z)$ in Eq. (3), it is trivial to show that an array factor of a metasurface or of an equivalent antenna array with a properly chosen phase distribution, $\psi_{l n}$, describes a desired discretized holographic mask. Indeed, a number of previous works have demonstrated successfully the use of metasurfaces for holography $[9,12,24]$. In the case of a perimeter-control architecture, phase $\psi_{l n}$ of the element in the $l$ th row and $n$th column is given by superposition of control signals $V_{l}$ and $U_{n}$ applied to respective columns and rows of the array. The array 
(a) $\quad \theta=30^{\circ}, \phi=0$

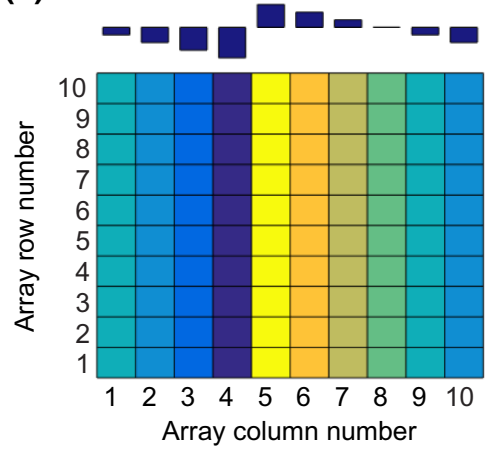

(b)

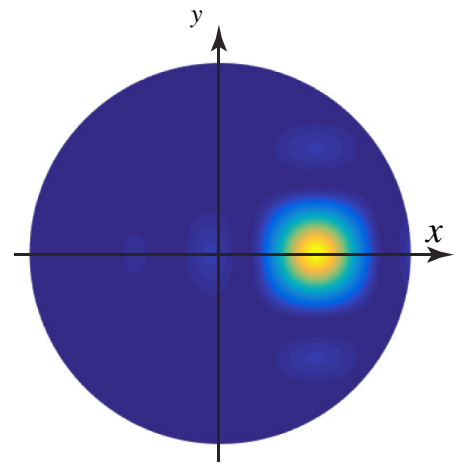

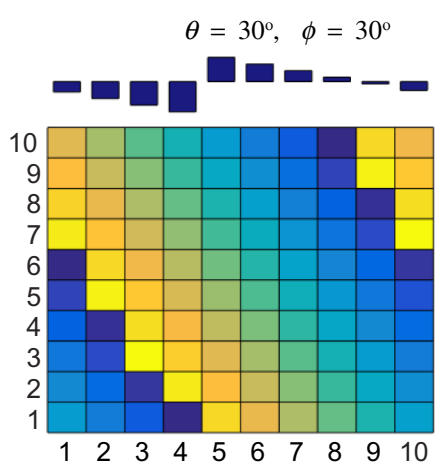
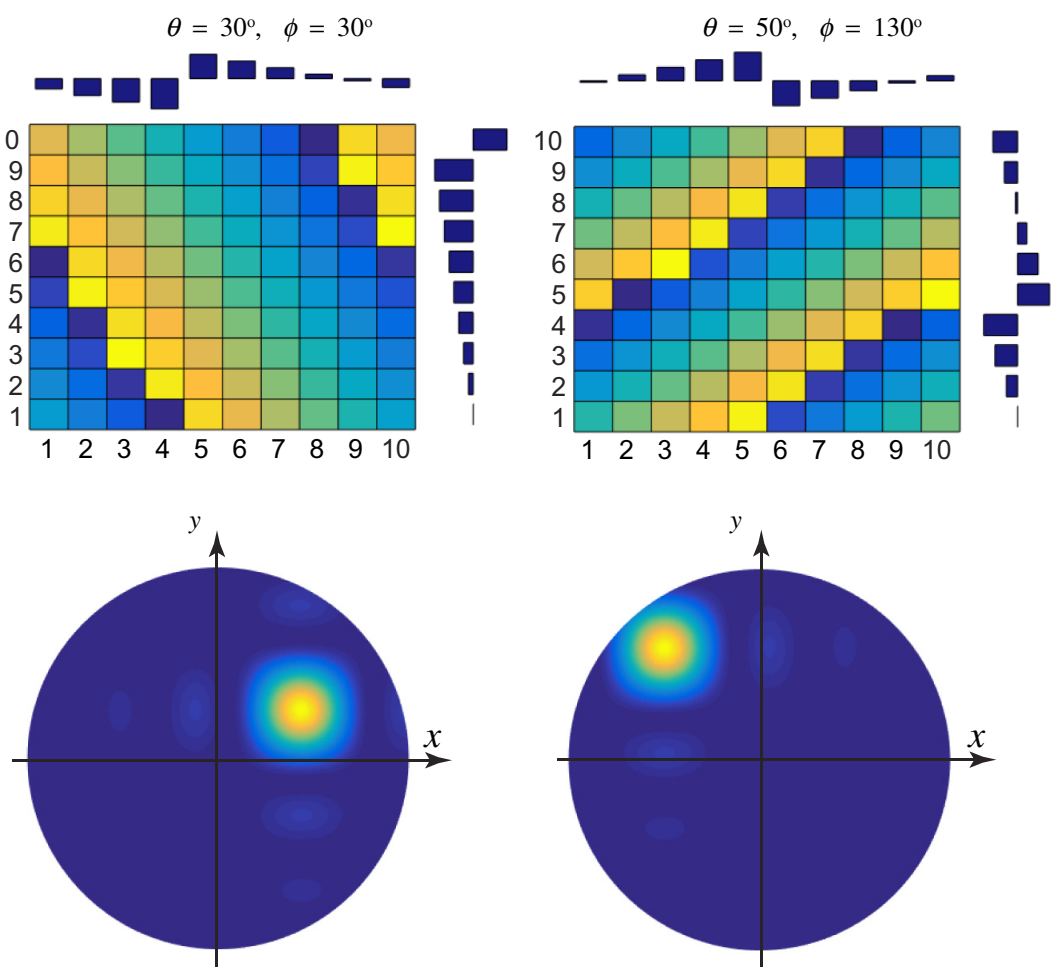

FIG. 2. Perimeter-controlled beam steering. (a) Distribution of phase across the array and respective control signals applied at the perimeter of a $10 \times 10$ array for several different target angles of beaming. (b) Respective radiation patterns in the far field.

factor in this case may be represented as

$$
\begin{aligned}
Z_{\text {perim }}(\theta, \phi) & \simeq \mathcal{F}_{2 \mathrm{D}}\left[\exp \left(i V_{l}+i U_{n}\right)\right] \\
& =\mathcal{F}_{2 \mathrm{D}}\left[e^{i V_{l}} \otimes e^{i U_{n}}\right]=I\left(x_{p}, y_{q}\right)
\end{aligned}
$$

Hence, our perimeter-control architecture can generate any phase mask that is given by an outer product of two linearly independent orthogonal vectors, i.e., $H_{\text {phase }}\left(x_{l}^{\prime}, y_{n}^{\prime}\right)=$ $e^{i V_{l}} \otimes e^{i U_{n}}$. Furthermore, since discrete Fourier transform is a linear operation, the reconstructed image may also be described as a product of two linearly independent functions, $f\left(x_{p}\right)$ and $g\left(y_{q}\right)$, i.e., $I\left(x_{p}, y_{q}\right)=f\left(x_{p}\right) \otimes g\left(y_{q}\right)$. Therefore, any image that can be represented as convolution of two orthogonal functions may be encoded by our perimeter-controlled holographic techniques.

To illustrate this, as an example, we consider HermitGaussian beams that can be represented as a product of two linearly independent functions. Specifically, the electric field at the waist is given as $E(x, y)=$ $H_{\eta_{x}}\left(\sqrt{2} x / w_{0}\right) e^{-x^{2} / w_{0}^{2}} H_{\eta_{y}}\left(\sqrt{2} y / w_{0}\right) e^{-y^{2} / w_{0}^{2}}$, where $H_{\eta}$ are $\eta$ th-order Hermit polynomials and $w_{0}$ is the beam waist. In Figs. 3(b)-3(d), we plot holographic reconstruction of a higher-order Hermite-Gaussian beam with the use of our technique. Here, we first create a discretized $512 \times 512$ pixel image, we then find a desired discrete holographic phase mask with the use of the commonly employed iterative Fourier transform algorithm [38]. We modify this algorithm to ensure that the generated discrete phase mask satisfies the condition of separability, i.e., $e^{i V_{l}} \otimes e^{i U_{n}}$. Clearly, the original image is fully reconstructed with our method.

Finally, we note that any complex image approximated by an $N \times N$ matrix, i.e., $I(x, y) \simeq I\left(x_{p}, y_{q}\right)$ with rank $\leq N$, with the use of a singular value decomposition may be represented as a sum of separable matrixes, $I\left(x_{p}, y_{q}\right)=\sum_{j} \hat{A}_{j}=\sum_{j} \sigma_{j} \mathbf{u}_{j} \otimes \mathbf{v}_{j}$, where $\mathbf{u}_{j}$ and $\mathbf{v}_{j}$ are the $j$ th columns of the corresponding singular value decomposed matrices, $\hat{A}_{j}$, and $\sigma_{j}$ are the ordered singular values. Clearly, our method allows encoding each of the $\hat{A}_{j}$ matrixes (here each of the matrices corresponds to a complex-valued scalar field). In most of practical cases, only $j=1, \ldots, M(M<N)$ of matrixes contribute to the formation of an image. All $N$ matrices are needed when all singular values of $\sigma_{j}$ are large. Figure 3(e) shows the application of our technique to holographic encoding and reconstruction of complex images. Here, an initial image, which is commonly used in computer vision algorithms, is, at first, factorized by singular value decomposition into a set of images described by separable matrices. Each of these "elemental" matrices can be holographically encoded with the help of our perimeter-control architecture [Fig. 3(c)]. The sum of elemental reconstructed images then approximates the original image. Projecting on a time-sharing basis, a final image (i.e., field) is formed with at most 
(a)
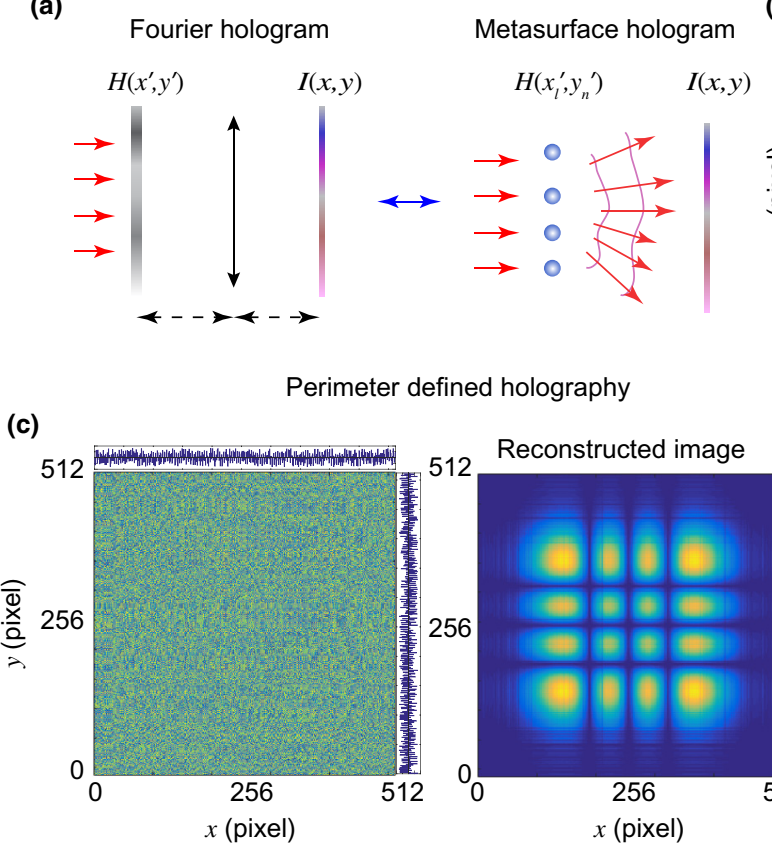

(b)

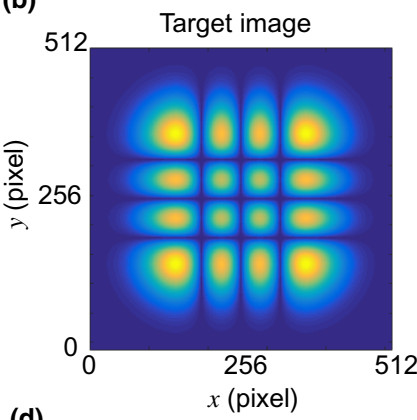

(d)

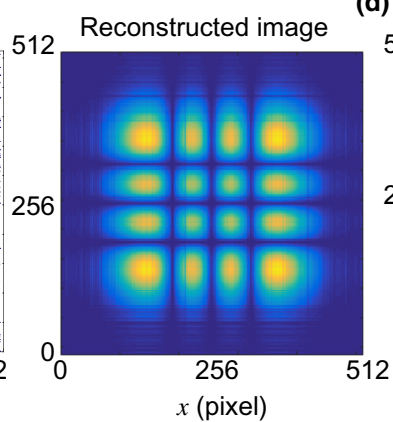

(e)

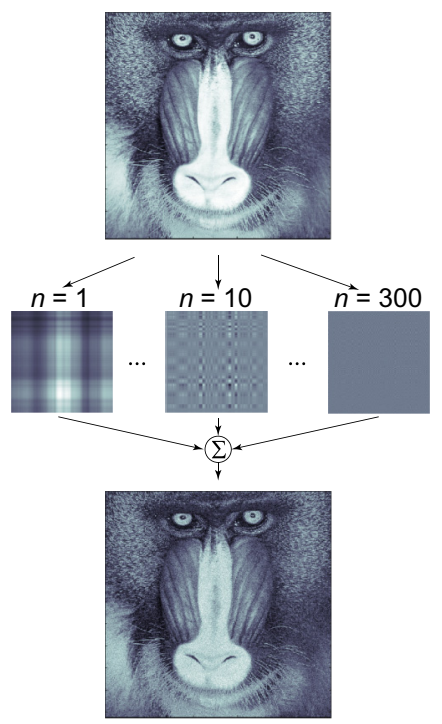

FIG. 3. (a) Principles of Fourier holography and its equivalence to metasurface-based image formation. (b) Original $512 \times 512$ pixel image of a $(3,3)$ Hermit-Gaussian mode. (c) Holographic reconstruction of the original image with perimeter-control architecture. Left panel shows phase distribution across the array, together with control signals applied to its rows and columns. Right panel shows a reconstructed image. Holographic image generated by an unconstrained array (i.e., phase of each element may be tuned independently) is shown for comparison in (d). (e) Application of perimeter-controlled holography to encoding of complex images. Full holographic image may be represented as a sum of simple images, each described by a separable matrix that can be encoded by our technique.

$M \ll N^{2}$ consecutive steps needed. In contrast, in raster scanning, an image is formed with $N \times N$ consecutive time steps (i.e., pixel by pixel projection).

\section{CONCLUSIONS}

Here, we study a case of phase-only modulation, assuming that the amplitude of emitters stays constant with an applied control signal. In practice, however, it is difficult to decouple amplitude and phase modulation [16,17]. At the same time, there is no constraint dictating that both amplitude and phase should vary with an applied control signal. In principle, a system with phase-only variation [15] may be designed. While, in the case of amplitude variation, the mathematical description developed earlier, strictly speaking, is not applicable, the perimeter-control architecture may be utilized to achieve functions discussed in the text with relevant optimization. This study, however, being specific to a given physical system, is outside the scope of this paper.

In conclusion, we introduce a perimeter-control architecture and show its use in a number of applications from beam steering with phased array antennas to holography with metasurfaces. Our approach is general and suggests an architecture that can be applied to a diverse set of physical arrays, including optical and microwave phased arrays, metasurfaces, and other arrayed systems.

\section{ACKNOWLEDGMENTS}

We are grateful for funding from Samsung Research America and UCLA startup funds.

\section{APPENDIX A: PHASE GRADIENT METASURFACE}

As discussed, the perimeter-control architecture can be utilized with phase gradient metasurfaces to control wave (beam) reflection and refraction. In this case, the direction of scattering may be controlled by inducing a desired phase gradient across the array. Figure 4 shows a schematic illustration of a metasurface with control signals applied along the perimeter.

\section{APPENDIX B: SCALING LAWS FOR OPTICAL PHASED ARRAYS AND METASURFACES}

Future applications of phased array optics, both antenna arrays and metasurfaces, would require large-aperture arrays to ensure long-range and efficient operation. In this section, we discuss the requirements for aperture size and the number of array elements.

In Fig. 5(a), we highlight several applications where optical phased arrays and metasurfaces are of particular interest. Specifically, a long-range operation is anticipated: from about $100 \mathrm{~m}$ for LIDARs on autonomous vehicles 


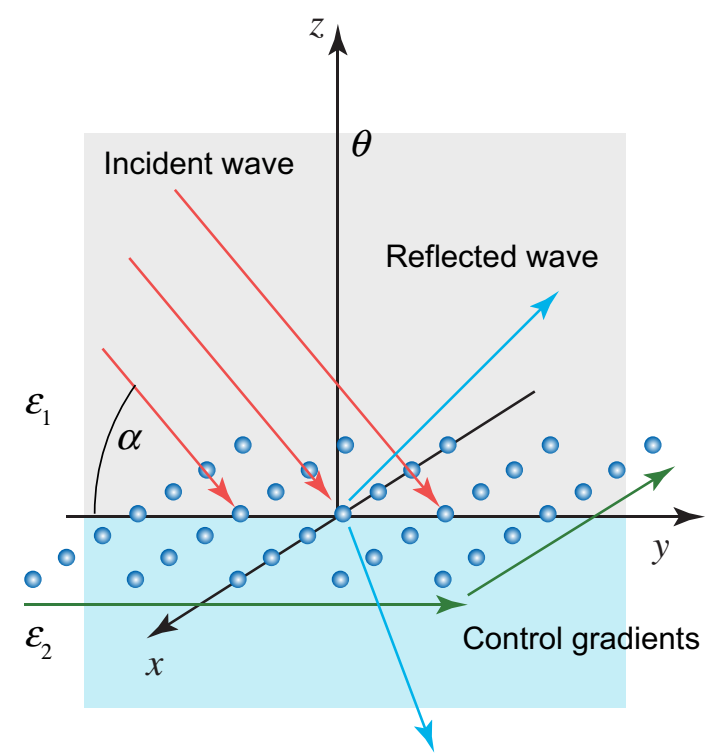

Refracted wave

FIG. 4. Schematic illustration of generalized laws of reflection and refraction with coherent phased arrays. Plane wave is incident at angle $\alpha$ on a planar array located at the interface of two media with permittivities $\varepsilon_{1}$ and $\varepsilon_{2}$. For small spacing between array elements and upon application of control signal gradients on the perimeter of the array, scattered reflected and refracted fields beyond conventional Snell's law are induced.

to over $100 \mathrm{~km}$ for space-based remote sensing and intersatellite communication systems. Let us consider a planar square antenna array with $N \times N$ elements [as shown schematically in Fig. 1(a)] with elements spaced $\lambda / 2$ away from each other. We shall now estimate the number $N$ that is needed for such long-range operation distances. We consider that the transmitter and receiver have similar antenna gain factors, $G \sim \pi N^{2}$. Using the Friis transmission formula, we estimate the number of antenna elements needed:

$$
G=\pi N^{2}=4 \pi \frac{d}{\lambda} \sqrt{\frac{P_{\min }}{P}},
$$

where $d$ is the distance between the transceiver and the receiver, $\lambda$ is the operation wavelength, $P$ is the power transmitted, and $P_{\min }$ is the minimum detectable signal at the receiver side.

To analyze Eq. (B1) further, it is convenient to express the detectable signal power through a signal-to-noise ratio, $P_{\min }=P_{\text {noise }} \delta$, where $P_{\text {noise }}$ is the noise power level and $\delta$ is the required signal-to-noise ratio. One of the key noise factors for such optical systems is the ambient solar radiation and albedo of the surroundings. We, hence, express the noise signal as $P_{\text {noise }}=I_{\text {ambient }} N^{2}(\lambda / 2)^{2}, I_{\text {ambient }} \simeq$ $A I_{\text {solar }}(\lambda) \Delta \lambda$, where $A$ is the albedo, $I_{\text {solar }}(\lambda)$ is the solar flux at wavelength $\lambda$, and $\Delta \lambda$ is the laser bandwidth. We may estimate the signal-to-noise ratio, $\delta$, from the Shannon's limit theorem as $\delta=\left[\left(\eta \Delta \lambda / \lambda^{2}\right) c\right]^{2}-1$, where $\eta$ is the desired acquisition rate for a LIDAR system, or a channel data rate for a communication system, and $c$ is the speed of light. Combining all of these expressions together, we find

$$
N^{2} \simeq 4 \frac{A I_{\text {solar }}(\lambda) \Delta \lambda}{P}\left[\left(\frac{\eta \Delta \lambda}{\lambda^{2}} c\right)^{2}-1\right] d^{2} .
$$

In Fig. 5(b), we plot the scaling law for the number of the array elements $\left(N^{2}\right)$ with the operation distance $d$ for some characteristic parameters. Specifically, we assume AM1.5 solar flux, a laser bandwidth of $\Delta \lambda=1 \mathrm{~nm}, \eta=1 \mathrm{GHz}$, and $P=1 \mathrm{~W}$. For these parameters, the signal-to-noise ratio is $\delta \sim 8$. According to this estimate, we find that for (a)

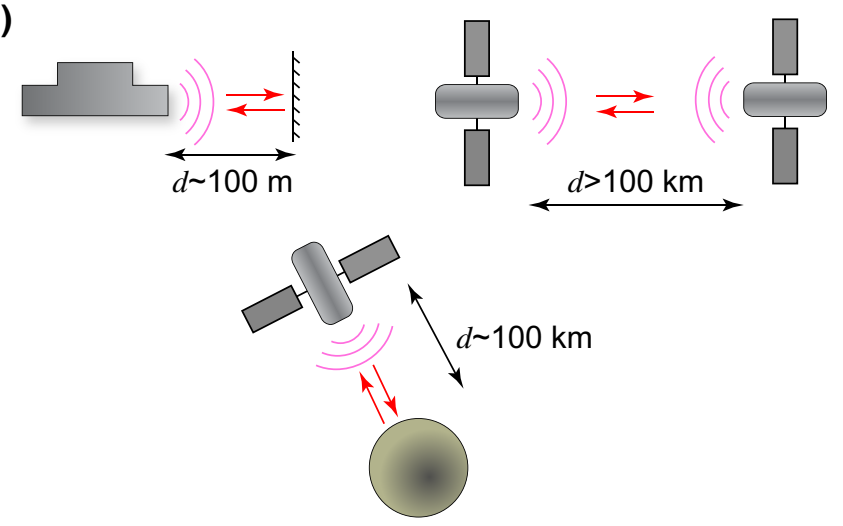

(b)

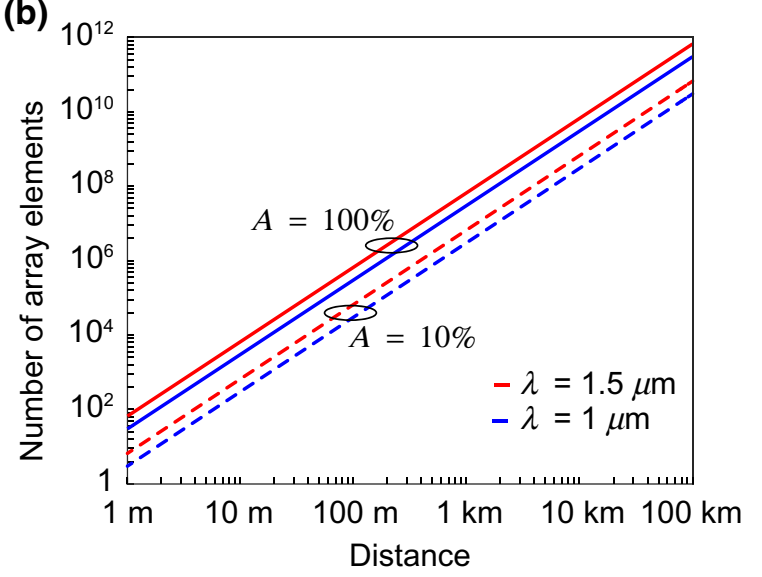

FIG. 5. Scaling of optical phased arrays. (a) Several typical applications where phased array optical antennas and metasurfaces may be of particular use, including LIDAR and space communications. Expected operation distances are shown schematically. (b) Number of array elements with the operation distance for two characteristic values of albedo and two wavelengths of operation. 
a $100 \mathrm{~m}$ operation distance an array with $10^{4}-10^{6}$ elements is needed, whereas for long-range space systems $10^{10}-10^{12}$ phase-tunable antenna elements are required! (We note that, despite a seemingly large number of elements, the characteristic aperture size is physically rather small: $\sim 1 \mathrm{~mm}$ and $\sim 10 \mathrm{~cm}$, respectively.) Clearly, addressing each and every element of the array individually represents a substantial challenge. The perimeter-control architecture discussed in the main text resolves this issue.

\section{APPENDIX C: REVIEW OF FUNDAMENTAL RADIATION PROPERTIES OF PHASED ARRAY ANTENNAS AND METASURFACES}

Here, we give a brief overview of key notions of radiation theory and its connection to phased array antennas, metasurfaces and diffraction theory. We first consider radiation properties of an individual source element, as shown schematically in Fig. 6. Such a source may be an element of an antenna array, a scatterer element of a metasurface, or an element of a diffractive optical system (e.g., hologram or phase grating). Without loss of generality, the properties of a source may be described by a current $\mathbf{J}\left(\mathbf{r}^{\prime}\right)=\mathbf{J}_{\text {source }}\left(\mathbf{r}^{\prime}\right)$, in the case of an antenna fed locally, or $\mathbf{J}\left(\mathbf{r}^{\prime}\right)=-i \omega\left[\mathbf{D}\left(\mathbf{r}^{\prime}\right)-\varepsilon_{0} \varepsilon \mathbf{E}\right]$, in the case of a scatterer, where the current is due to the polarization density induced in the scatterer by an incident field $\mathbf{E}_{\text {inc }}$ (Fig. 6). The radiated field in the far zone (i.e., for $\mathbf{k r} \rightarrow \infty$, where $\mathbf{k}$ is the free space wavevector in the direction of the observation point $P$ ) may be expressed with the use of a magnetic vector potential field $\mathbf{A}(\mathbf{r})$ as $[1,37,39]$

$$
\begin{aligned}
\mathbf{A}(\mathbf{r}) & =\frac{\mu_{0}}{4 \pi} \lim _{k r \rightarrow \infty} \int \mathbf{J}\left(\mathbf{r}^{\prime}\right) \frac{e^{i k\left|\mathbf{r}-\mathbf{r}^{\prime}\right|}}{\left|\mathbf{r}-\mathbf{r}^{\prime}\right|} d^{3} \mathbf{r}^{\prime} \\
& =\frac{\mu_{0}}{4 \pi} \frac{e^{i k r}}{r} \int \mathbf{J}\left(\mathbf{r}^{\prime}\right) e^{-i \mathbf{k r}^{\prime}} d^{3} \mathbf{r}^{\prime}
\end{aligned}
$$

Here, $\mu_{o}$ is the free-space permeability, integration is over the volume of the source $\mathbf{J}\left(\mathbf{r}^{\prime}\right)$, and $\mathbf{r}^{\prime}$ is the radius vector of the source. Notably, we use a common approximation that assumes $\left|\mathbf{r}-\mathbf{r}^{\prime}\right| \simeq r$ and $e^{i k\left|\mathbf{r}-\mathbf{r}^{\prime}\right|} \simeq e^{i k r} e^{-i k r^{\prime}}$, physically corresponding to the case of observation distances much larger than the dimensions of an optical system. It is convenient to rewrite Eq. (C1) with respect to the center of the source $\mathbf{r}_{0}=\mathbf{r}^{\prime}-\boldsymbol{\xi}$ (see also Fig. 6):

$$
\mathbf{A}(r)=\frac{\mu_{0}}{4 \pi} \frac{e^{i k r}}{r} e^{-i k r_{0}} \int \mathbf{J}(\boldsymbol{\xi}) e^{-i \mathbf{k} \xi} d^{3} \boldsymbol{\xi}
$$

At the limit of a source smaller than the wavelength, i.e., when $d \leq \lambda$, the integrand may be expanded into a series with first-order responses given by excited dipole moments (we neglect the contribution of an electric quadrupole

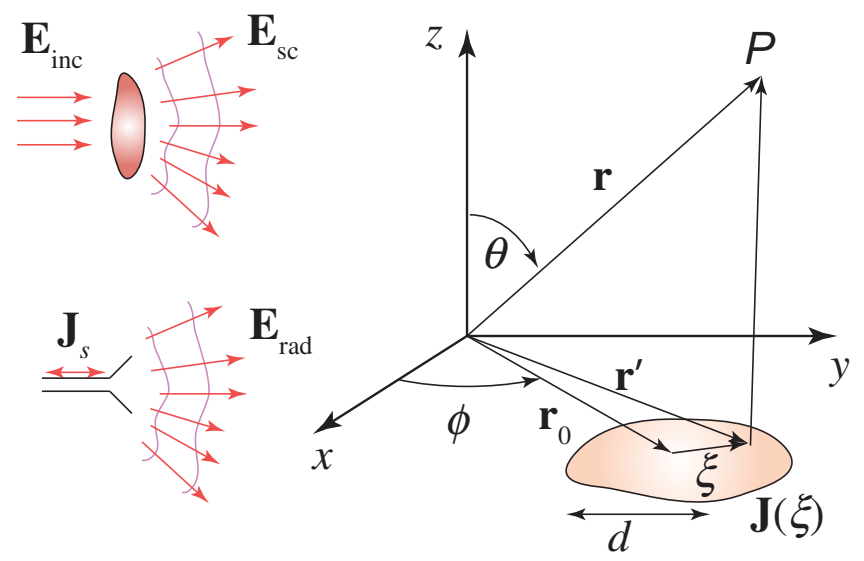

FIG. 6. Schematic illustration of a geometry studied. Difference between antenna and scatterer is also shown.

here):

$$
\mathbf{A}(r)=\frac{\mu_{0}}{4 \pi} \frac{e^{i k r}}{r} e^{-i \mathbf{k r}_{0}}[-i \omega \mathbf{p}+i(\mathbf{k} \times \mathbf{m})]
$$

where $\quad \mathbf{p}=(1 / i \omega) \int \xi \nabla \mathbf{J}(\boldsymbol{\xi}) d^{3} \boldsymbol{\xi}$ and $\quad \mathbf{m}=1 / 2 \int[\boldsymbol{\xi} \times$ $\mathbf{J}(\xi)] d^{3} \boldsymbol{\xi}$ are electric and magnetic dipole moments, respectively. For the case of a metasurface consisting of small scatters, these dipole moments may be expressed as $\mathbf{p}=\overline{\bar{\alpha}}_{e} \mathbf{E}_{\text {inc }}$ and $\mathbf{m}=\overline{\bar{\alpha}}_{m} \mathbf{E}_{\text {inc }}$, where $\overline{\bar{\alpha}}_{e}$ and $\overline{\bar{\alpha}}_{m}$ are electric and magnetic polarizability tensors, respectively.

The electric field at point $\mathbf{r}$ is then found to be $\mathbf{E}(r)=$ $(i c / k) \nabla \times \nabla \times \mathbf{A}(\mathbf{r})$ and at the limit of $k r \rightarrow \infty$ and $r \gg$ $r_{0}$ it is given as

$$
\mathbf{E}(\mathbf{r})=e^{-i \mathbf{k r}_{0}}\left\{\left[\frac{k \mathbf{m}}{c}+(\mathbf{k} \times \mathbf{p})\right] \times \mathbf{k}\right\} \frac{e^{i \mathbf{k r}}}{4 \pi \varepsilon_{0} r} .
$$

Notably, in the case of a scatterer, $\mathbf{E}$ denotes the scattered field $\mathbf{E}=\mathbf{E}_{\mathrm{sc}}$, and the total field is given as a superposition of the incident wave and the scattered wave, $\mathbf{E}_{\text {total }}=$ $\mathbf{E}+\mathbf{E}_{\text {inc }}$. In Eq. (C4), the first term is associated with the location of the source and corresponds to the geometric phase delay, whereas the term in parentheses denotes the intrinsic radiation properties of the source, commonly denoted as an antenna factor in the context of phased array antennas. It is convenient to represent the latter as $\mathbf{a} e^{i \psi}=\{[(k / c) \mathbf{m}+(\mathbf{k} \times \mathbf{p})] \times \mathbf{k}\}\left(e^{i \mathbf{k r}} / 4 \pi \varepsilon_{0} r\right)$, so that the expression for the electric field assumes a compact form:

$$
\mathbf{E}=e^{-i \mathbf{k} \mathbf{r}_{0}} \mathbf{a} e^{i \psi},
$$

where a and $\psi$ correspond to the amplitude and phase of the source, respectively. 


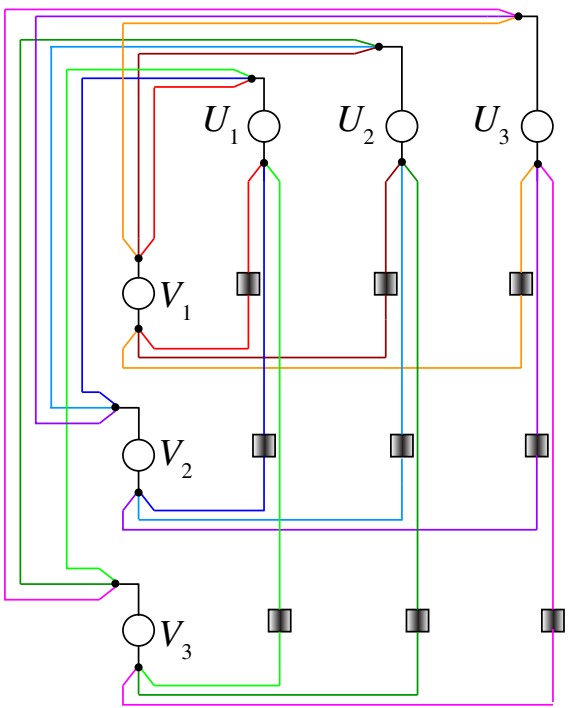

FIG. 7. Schematic illustration of a circuit layout for a perimeter-control architecture. Perimeter-control architecture for a $3 \times 3$ array. Colors encode independent circuit loops. Points of common contact are shown as dots. Circles denote voltage sources placed on the perimeter of the array and shaded boxes correspond to array emitters.

\section{APPENDIX D: CIRCUIT IMPLEMENTATION OF DC VOLTAGE CONTROLLED ARRAY}

In the architecture we propose, we assume that signals applied at each row and column of the array are superimposed at a corresponding array element, i.e., $V_{v}=f\left(V_{l}+\right.$ $\left.U_{n}\right) ; V_{l}$ and $U_{n}$ denote control signals applied to column $l$ and row $n$ of the array, respectively. Our approach is applicable to a variety of control methods, including temperature, electrical signal, and magnetic bias, as soon as the superposition principle works for control signals.

Direct electrical control of the emitter properties with an applied bias voltage is of great interest [17]. In this case, a dc voltage-controlled circuit may be implemented via voltage sources connected in series. It is important to ensure that each of the array elements sees only two of the required voltage sources connected in series. Figure 7 shows a schematic layout of control architecture for a $3 \times 3$ array. In this architecture, each of the array elements is fed by an independent circuit comprised of two respective voltage sources. The voltage drop across the element, by Kirchhoff's law, is equal to the sum of voltages. Notably, circuits with individual antenna elements are independent of each other.

[1] C. A. Balanis, Antenna Theory: Analysis and Design, 3rd Ed. (Wiley-Interscience, Hoboken, 2005).
[2] J. Sun, E. Timurdogan, A. Yaacobi, E. S. Hosseini, and M. R. Watts, Large-scale nanophotonic phased array, Nature 493, 195 (2013).

[3] F. Aflatouni, B. Abiri, A. Rekhi, and A. Hajimiri, Nanophotonic projection system, Opt. Express 23, 21012 (2015).

[4] C. V. Poulton, A. Yaacobi, D. B. Cole, M. J. Byrd, M. Raval, D. Vermeulen, and M. R. Watts, Coherent solid-state LIDAR with silicon photonic optical phased arrays, Opt. Lett. 42, 4091 (2017).

[5] K. Sayyah, O. Efimov, P. Patterson, J. Schaffner, C. White, J.-F. Seurin, G. Xu, and A. Miglo, Two-dimensional pseudo-random optical phased array based on tandem optical injection locking of vertical cavity surface emitting lasers, Opt. Express 23, 19405 (2015).

[6] D. N. Hutchinson, J. Sun, J. K. Doylend, R. Kumar, J. Heck, W. Kim, C. T. Phare, A. Feshali, and H. Rong, Highresolution aliasing-free optical beam steering, Optica 3, 887 (2016).

[7] J. K. Doylend, M. J. R. Heck, J. T. Bovington, J. D. Peters, L. A. Coldren, and J. E. Bowers, Two-dimensional freespace beam steering with an optical phased array on siliconon-insulator, Opt. Express 19, 21595 (2011).

[8] D. Kwong, A. Hosseini, J. Covey, Y. Zhang, X. Xu, H. Subbaraman, and R. T. Chen, On-chip silicon optical phased array for two-dimensional beam steering, Opt. Lett. 39, 941 (2014).

[9] N. Yu and F. Capasso, Flat optics with designer metasurfaces, Nat. Mater. 13, 139 (2014).

[10] Z. Zhu, P. G. Evans, R. F. Haglund, and J. G. Valentine, Dynamically reconfigurable metadevice employing nanostructured phase-change materials, Nano Lett. 17, 4881 (2017).

[11] G. Kafaie-Shirmanesh, R. Sokhoyan, R. A. Pala, and H. A. Atwater, Dual-Gated active metasurface at $1550 \mathrm{~nm}$ with wide $\left(>300^{\circ}\right)$ phase tunability, Nano Lett. 18, 2957 (2018).

[12] G. Zheng, H. Mühlenbernd, M. Kenney, G. Li, T. Zentgraf, and S. Zhang, Metasurface holograms reaching $80 \%$ efficiency, Nat. Nanotechnol. 10, 308 (2015).

[13] Q. He, S. Sun, and L. Zhou, Tunable/reconfigurable metasurfaces: Physics and applications, Research 2019, 1849272 (2019).

[14] A. Howes, W. Wang, I. Kravchenko, and J. Valentine, Dynamic transmission control based on all-dielectric Huygens metasurfaces, Optica 5, 787 (2018).

[15] S.-Q. Li, X. Xu, R. M. Veetil, V. Valuckas, R. PaniaguaDomínguez, and A. I. Kuznetsov, Phase-only transmissive spatial light modulator based on tunable dielectric metasurface, Science 364, 1087 (2019).

[16] P. C. Wu, R. A. Pala, G. Kafaie-Shirmanesh, W.-H. Cheng, R. Sokhoyan, M. Grajower, M. Z. Alam, D. Lee, and H. A. Atwater, Dynamic beam steering with all-dielectric electro-optic III-V multiple-quantum-well metasurfaces, Nat. Commun. 10, 1 (2019).

[17] G. Kafaie-Shirmanesh, R. Sokhoyan, P. C. Wu, H.A. Atwater "Electro-Optically Tunable Universal Metasurfaces," arXiv:1910.02069 (2019).

[18] A. L. Holsteen, A. F. Cihan, and M. L. Brongersma, Temporal color mixing and dynamic beam shaping with silicon metasurfaces, Science 365, 257 (2019). 
[19] A. M. Shaltout, V. M. Shalaev, and M. L. Brongersma, Spatiotemporal light control with active metasurfaces, Science 364, 6441 (2019).

[20] P. P. Iyer, M. Pendharkar, C. J. Palmstrøm, and J. A. Schuller, III-V heterojunction platform for electrically reconfigurable dielectric metasurfaces, ACS Photonics 6, 1345 (2019).

[21] M. J. R. Heck, Highly integrated optical phased arrays: Photonic integrated circuits for optical beam shaping and beam steering, Nanophotonics 6, 152 (2017).

[22] W. M. Neubert, K. H. Kudielka, W. R. Leeb, and A. L. Scholtz, Experimental demonstration of an optical phased array antenna for laser space communications, Appl. Opt. 33, 3820 (1994).

[23] S. Serati and J. Stockley, Phased array of phased arrays for free space optical communications, IEEE Aerosp. Conf. Proc. 4, 1085 (2003).

[24] G.-Y. Lee, J. Sung, and B. Lee, Recent advances in metasurface hologram technologies (Invited paper), ETRI J. 41, 10 (2019).

[25] D. Fattal, Z. Peng, T. Tran, S. Vo, M. Fiorentino, J. Brug, and R. G. Beausoleil, A multi-directional backlight for a wide-angle, glasses-free three-dimensional display, Nature 495, 348 (2013).

[26] N. Ojaroudiparchin, M. Shen, S. Zhang, and G. F. Pedersen, A switchable 3-D-coverage-phased array antenna package for 5G mobile terminals, IEEE Antenna Wirel. Lett. 15, 1747 (2016).

[27] B. Sadhu, et al., in 2017 IEEE International Solid-State Circuits Conference (ISSCC), San Francisco, CA, (2017).

[28] "Millimeter-Wave Circuits for $5 G$ and Radar" Ed. G. Hueber, A. M. Niknejad (Cambridge University Press, 2019).
[29] A. H. Naqvi and S. Lim, Review of recent phased arrays for millimeter-wave wireless communication, Sensors 18, 3194 (2018).

[30] V. F.-G. Tseng, S. S. Bedair, and N. Lazarus, Phased array focusing for acoustic wireless power transfer, IEEE Trans. Ultrason., Ferroelectr., Freq. Control 65, 39 (2018).

[31] A. Massa, G. Oliveri, F. Viani, and P. Rocca, Array designs for long-distance wireless power transmission: State-ofthe-Art and innovative solutions, Proc. IEEE 101, 1464 (2013).

[32] A. She, S. Zhang, S. Shian, D. R. Clarke, and F. Capasso, Large area metalenses: Design, characterization, and mass manufacturing, Opt. Express 26, 1573 (2018).

[33] R. J. Rogorzelski, On the dynamics of two-dimensional array beam scanning via perimeter detuning of coupled oscillator arrays, IEEE Trans. Antennas Propag. 49, 234 (2001).

[34] C. Carta, M. Seo, and M. Rowell, A mixed-signal row/column architecture for very large monolithic mmwave phased arrays, Int. J. High Speed Electron. Syst. 17, 01 (2007).

[35] M. Sanchez-Barbetty and R. W. Jackson, in 2008 IEEE MTT-S International Microwave Symposium Digest (2008).

[36] F. Ashtiani and F. Aflatouni, $\mathrm{N} \times \mathrm{N}$ optical phased array with 2N phase shifters, Opt. Express 19, 27183 (2019).

[37] M. Born and E. Wolf, Principles of Optics - 7th ed. (Cambridge University Press, Cambridge, 1980).

[38] T. C. Poon and J. P. Liu, Introduction to Modern Digital Holography with Matlab (Cambridge University Press, New York, 2014).

[39] L. Novotny and B. Hecht, Principles of Nano-Optics (Cambridge University Press, New York, 2006). 